TRANSACTIONS OF THE AMERICAN MATHEMATICAL SOCIETY

Volume 348, Number 11, November 1996

\title{
THE STABILITY OF FOLIATIONS OF ORIENTABLE 3-MANIFOLDS COVERED BY A PRODUCT
}

\author{
SANDRA L. SHIELDS
}

\begin{abstract}
We examine the relationship between codimension one foliations that are covered by a trivial product of hyperplanes and the branched surfaces that can be constructed from them. We present a sufficient condition on a branched surface constructed from a foliation to guarantee that all $C^{1}$ perturbations of the foliation are covered by a trivial product of hyperplanes. We also show that a branched surface admits a strictly positive weight system if and only if it can be constructed from a fibration over $S^{1}$.
\end{abstract}

\section{INTRODUCTION}

In this paper we consider transversely orientable codimension one $C^{1}$ foliations of smooth Riemannian 3-manifolds. We address a question posed by W. Thurston: Is the property of being covered by the canonical foliation of $\mathfrak{R}^{3}$ by parallel hyperplanes stable under $C^{1}$ perturbations of the foliation? We use branched surface constructed from foliations to find a sufficient condition to guarantee this stability.

The idea of using branched surfaces to study foliations can be traced back to Williams ([16]). In [1] Christy and Goodman describe a technique for constructing a branched surface from any transversely oriented codimension one foliation of a 3manifold. The branched surface $W$, which gives a skeletal outline of the foliation, is imbedded in the 3-manifold and is said to "carry" the foliation. The foliation can be retrieved by foliating a neighborhood $N(W)$ of $W$ in a particular way and collapsing the complement of this neighborhood. Other foliations of this neighborhood yield foliations of the 3-manifold which are also carried by the branched surface. For a foliation $f$ of a closed manifold, a branched surface constructed from $f$ carries all sufficiently small $C^{1}$ perturbations of $f$ (i.e. foliations sufficiently close to $f$ in the $C^{1}$ metric defined by Hirsch in [6]). So to obtain stability results, we look for properties of the branched surface that imply something about the qualitative structure of the foliations carried by it. It is important to note that foliations that are not $C^{1}$ perturbations of $f$ may be carried by the same branched surface as $f$. So these properties guarantee a certain structure for a broader range of foliations than those obtained by perturbing $f$ slightly.

In $\S I I$ we review branched surfaces imbedded in 3-manifolds that are constructed using Christy and Goodman's technique. These branched surfaces are the same as

Received by the editors February 5, 1993 and, in final form, October 9, 1995.

1991 Mathematics Subject Classification. Primary 57M12, 57M20, 57N10, 57R30, 58F10.

Key words and phrases. Branched surface, foliation, leaf space, holonomy map, topological equivalency. 
those constructed in [3] from laminations of 3-manifolds. Therefore, they are more restricted than those in [16]. Since the construction is not essential to understanding the results of this paper, we omit it here and refer the reader to [1]. Instead, we review important properties of this construction and outline the relationship between a branched surface and the foliations it carries.

It is well known that fibrations over $S^{1}$ are covered by a trivial product of hyperplanes (that is, a foliation topologically equivalent to the canonical foliation of $\mathfrak{R}^{3}$ by parallel hyperplanes) when the fiber is not $S^{2}$. In $\S I I I$ we show that a compact branched surface carries a fibration over $S^{1}$ if and only if it admits a strictly positive weight system. We show how to use a strictly positive weight system on a branched surface to determine the genus of the fiber. We then apply a result due to Gabai which states that if the genus of the fiber is greater than one, then the foliation by fibers is unstable under $C^{0}$ perturbations (using the $C^{0}$ metric of [6]). That is, a foliation can be constructed which is $C^{0}$ close to the fibration but has no compact leaves. The branched surface carries both foliations.

In $\S I V$, we show that the existence of a curve in $\mathfrak{R}^{3}$ that satisfies a simple relationship with the covering of a compact branched surface is enough to guarantee that every foliation carried by the branched surface has a covering by a trivial product of hyperplanes. We use this to find a sufficient condition on a branched surface constructed from a foliation covered by a trivial product of hyperplanes to ensure that all sufficiently small $C^{1}$ perturbations of the foliation are also covered by a trivial product of hyperplanes.

The author would like to thank Sue Goodman for many conversations and Bev Diamond for numerous suggestions which improved the manuscript.

\section{BRANChED SURFACES TRANSVERSE TO A FLOW}

Throughout this paper, $M$ will be a smooth Riemannian 3-manifold without boundary. We consider only those branched surfaces that can be constructed from a codimension one $C^{1}$ foliation using the technique described by Christy and Goodman. For this reason, our branched surfaces will be more restricted than those considered in [16].

For Christy and Goodman's construction, we choose a flow $\phi$ transverse to the foliation $f$ and a "generating set" $\Delta=\left\{D_{\alpha}\right\}_{\alpha \in A}$ consisting of imbedded disjoint compact planar surfaces that are contained in leaves and satisfy certain general position requirements with respect to $\phi$. If $M$ is compact, we require $\Delta$ to be finite; this ensures the branched surface is compact.

We take the complement of $\bigcup_{\alpha \in A} \operatorname{int}\left(D_{\alpha}\right)$ in $M$. It is homeomorphic to a connected subset $M^{*}$ of $M$ whose complement is the union of compact 3-manifolds and whose closure is a manifold $N(W)$ with a furrowed boundary. That is, $N(W)$ is a space with a $C^{1}$ atlas with three sorts of local models: neighborhoods in a 3-dimensional Euclidean space, neighborhoods in a 3-dimensional Euclidean halfspace, and neighborhoods of the origin in the product of $\left\{(x, y)\right.$ in $\mathfrak{R}^{2} \mid x<0$, or $y \geq x^{2}$, or $\left.y \leq-x^{2}\right\}$ with a 1-dimensional Euclidean space. Boundary points of $N(W)$ with a local neighborhood modeled on the latter type are called "furrow points" of $N(W)$. We think of $N(W)$ as the space we obtain when we cut $M$ open along the elements in $\Delta$. The inverse homeomorphism from $M^{*}$ onto $M-\bigcup_{\alpha \in A} \operatorname{int}\left(D_{\alpha}\right)$ extends to a map $h: M \rightarrow M$. So there is a flow $\phi^{*}$ on $M$ that is transverse to $\partial N(W)$ and restricts to a flow on $M^{*}$ which is topologically 

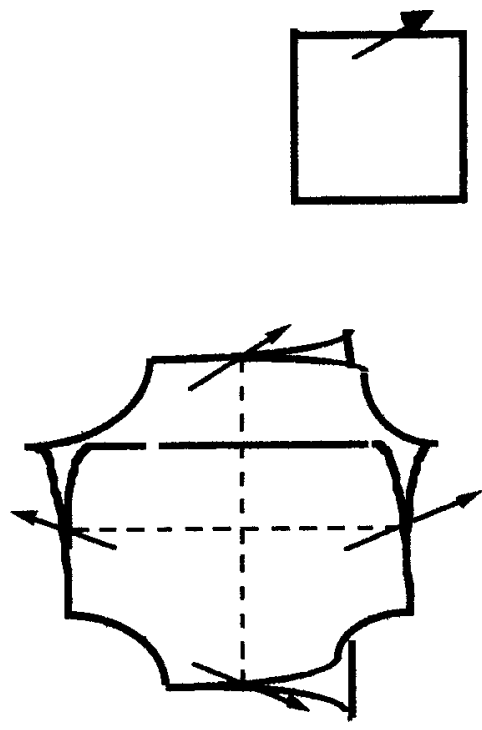

FiguRE 2.2

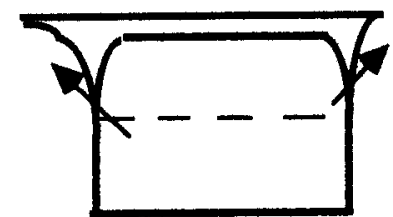

Figure 2.1

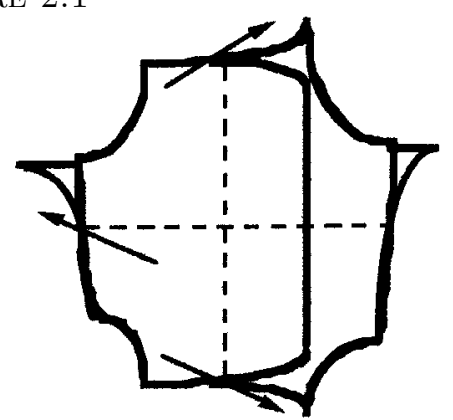

FIGURE 2.3

equivalent, via $h$, to the restriction of $\phi$ to $M-\bigcup_{\alpha \in A} \operatorname{int}\left(D_{\alpha}\right)$. The flow $\phi^{*}$ defines a transverse orientation on $\partial N(W)$. The furrow points of $N(W)$ divide each component of $\partial N(W)$ into two homeomorphic surfaces: one with an outwardly directed transverse orientation and the other with an inwardly directed transverse orientation. We refer to these surfaces as the "lower" and "upper" hemisphere, respectively, of this component.

One of the general position requirements for elements of the generating set $\Delta$ is that every orbit of $\phi$ meets the interior of some element of $\Delta$ in forward and backward time. So the restriction of each orbit of $\phi^{*}$ to $N(W)$ is the union of disjoint segments, each homeomorphic to the unit interval, which we call "fibers" of $N(W)$. We use these fibers to define an equivalence relation on $N(W)$, where $x \sim y$ if $x$ and $y$ lie on the same fiber. We then construct the quotient space $N(W) / \sim$, identifying each fiber to a point.

We obtain an imbedding of $N(W) / \sim$ that is transverse to $\phi^{*}$ by expanding the components of $M-N(W)$ until each fiber is contracted to a point (i.e. we collapse $N(W)$ along the interval orbits of $\left.\left.\phi^{*}\right|_{N(W)}\right)$. This imbedding is the branched surface $W$. It has a smooth structure induced by $N(W)$ and a transverse orientation induced by $\phi^{*}$. Clearly, we may thicken $W$ along segments of orbits of $\phi^{*}$ to retrieve $N(W)$.

It is worth noting that a branched surface may be constructed from any foliation and transverse flow. More precisely, there is always a set of disks imbedded in leaves of the foliation that satisfy the general position requirements for a generating set with respect to the transverse flow. For simplicity, we will assume the surfaces in the generating set are imbedded disks unless otherwise stated. Nonetheless, the results in this paper hold whenever the generating set is a collection of imbedded compact planar surfaces.

The disks in $\Delta$ are chosen so that $W$ is a connected 2-dimensional complex with a set of charts defining orientation-preserving local diffeomorphisms onto one of the models in Figure 2.1, 2.2, or 2.3 such that the transition maps are smooth and pre- 


\section{Local W}

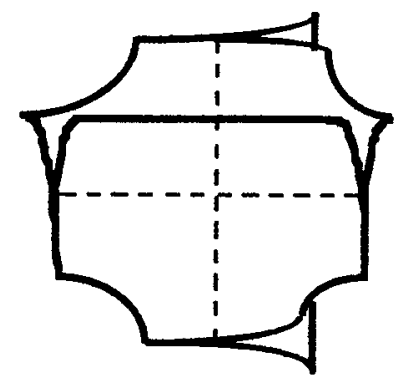

S. L. SHIELDS

\section{Thicken to local N(W):}

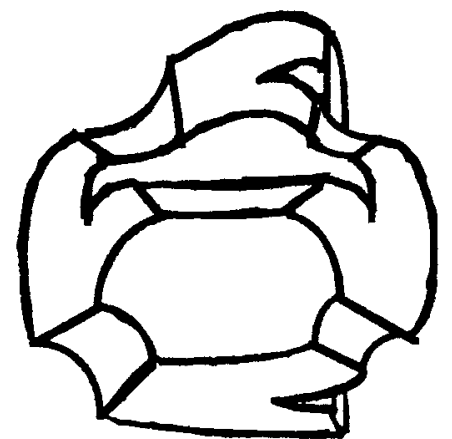

FiguRE 2.4

serve the transverse orientation indicated by the arrows. Each local model projects horizontally onto a vertical model of $\mathfrak{R}^{2}$; therefore, $T \mathfrak{R}^{2}$ induces the tangent bundle on $W$ when we pull back each local projection. An analytic description of the models can be found in [2].

Each branched surface $W$ is a connected 2-manifold except on a small subset $B$ called the "branch set." A point in the branch set is called a "branch point". The elements of the generating set $\Delta$ can be chosen large enough to ensure $B$ is connected, so we will always assume this to be the case. The set $B$ is a 1-manifold except at isolated points called "crossings". A "right" ("left", respectively) crossing has a local neighborhood that is mapped by a chart onto the model in Figure 2.2 (Figure 2.3, respectively). Figure 2.4 shows a local neighborhood in $N(W)$ obtained by thickening a neighborhood of a right crossing along segments of orbits of $\phi^{*}$. For $x \in W$, we say the fiber obtained by thickening $x$ lies "over" $x$ in $N(W)$. Accordingly, each point in this fiber is "over" $x$. (We note that this is a slight abuse of terminology, since $N(W)$ is not quite an interval fiber bundle over $W$.) For each connected subset $K$ of $W$, we use $N(K)$ to denote the submanifold of $N(W)$ consisting of the union of fibers over points in $K$.

Formally, a "curve" in $M$ is a continuous map from a connected subset of $\mathfrak{R}$ into $M$. However, we consider a curve to be the image of such a map, where the map parameterizes the curve. The "beginning" and "end" of a curve refer to the negative and positive boundary, respectively, induced by the parameterization. For the sake of simplicity, we consider two curves $\gamma$ and $\gamma^{\prime}$ to be the same if $\gamma(t)=\gamma^{\prime}(t)$ after some orientation-preserving reparameterization of each. A curve $\gamma^{\prime}$ is contained in a second curve $\gamma$ if there exist curves $\alpha_{1}$ and $\alpha_{2}$ such that $\gamma$ is equal to the composition $\alpha_{2} * \gamma^{\prime} * \alpha_{1}$.

The connected components of $W-B$ are called the "sectors of $W$ ". It is worth noting that these sectors are imbedded planar surfaces, since the generating set for $W$ consists of imbedded disks (and annuli in the more general case). At any point $x \in B$ that is not a crossing, there are locally 3 adjacent sectors, $S_{1}, S_{2}$, and $S_{3}$, such that $\operatorname{cl}\left(S_{1}\right) \cup \operatorname{cl}\left(S_{2}\right)$ and $\operatorname{cl}\left(S_{2}\right) \cup \operatorname{cl}\left(S_{3}\right)$ are contained in smooth submanifolds of $W$ (i.e. the set of charts locally defines a smooth immersion into a planar subset of $\mathfrak{R}^{3}$ on $\operatorname{cl}\left(S_{1}\right) \cup \operatorname{cl}\left(S_{2}\right)$ and $\left.\operatorname{cl}\left(S_{2}\right) \cup \operatorname{cl}\left(S_{3}\right)\right)$. We say $S_{1}$ and $S_{3}$ are "tangent" to 
$S_{2}$ at $x$. Suppose in a local neighborhood of $x$, forward orbits under $\phi^{*}$ of points in $S_{1}$ flow into $S_{3}$. Then, for any nontrivial curve $\beta$ that begins (or ends) at $x$ and contains an arc in $S_{2}$ which also begins (respectively, ends) at $x$, we say $S_{3}$ is the "upper" sector branching from $\beta$ at $x$ and $S_{1}$ is the "lower" sector branching from $\beta$ at $x$. Given a curve $\alpha$ containing $\beta$, any sectors branching from the beginning of $\beta$ are "incoming" sectors along $\alpha$ and any sectors branching from the end of $\beta$ are "outgoing" sectors along $\alpha$.

At times it will be convenient to represent only a regular neighborhood $W_{\varepsilon}$ of the branch set $B$ in $W$. For this we use the graph of $B$ with a neighborhood of each crossing imbedded in the plane and labeled " $\mathrm{L}$ " or " $\mathrm{R}$ " to indicate left or right respectively. The position of the label indicates the 3 -sheeted side of the branch set. The edges between crossings of $B$ describe the transition maps (i.e. these edges describe the manner in which we piece together local neighborhoods of the crossings in $W$, by pairwise identifying the Y-shaped components of their boundaries, to obtain $W_{\varepsilon}$ ). The branched surface $W$ is represented by this labeled graph together with a description of its sectors.

Foliations carried by branched surfaces. We consider only foliations of $N(W)$ by leaves (possibly branched) that are transverse to the fibers of $N(W)$. We require that each component of $\partial N(W)$ is contained in a leaf and that the set of branch points for the leaves is the same as the set of furrow points in $\partial N(W)$. A "foliation" of $N\left(W_{\varepsilon}\right)$ is defined similarly and is everywhere transverse to $\partial N\left(W_{\varepsilon}\right)$ except where it intersects $\partial N(W)$. (See Figure 2.5.) It is important to note that a foliation of $N\left(W_{\varepsilon}\right)$ does not necessarily extend to a foliation of $N(W)$, since there is the possibility that extending may force nontrivial holonomy along $\partial N(S)$ for some disk sector $S$.

When we cut the manifold $M$ open along the disks in the generating set of $W$, the foliation $f$ transverse to the flow $\phi$ gives rise to a unique foliation $f^{*}$ of $N(W)$ which is transverse to the induced flow $\phi^{*}$. Conversely, we recover $f$ when we "collapse" the components of $\overline{M-N(W)}$ by identifying points that lie on the same segment of orbit of $\left.\phi^{*}\right|_{M-N(W)}$. Moreover, any foliation of $N(W)$ yields a foliation of $M$, which we say is "carried" by $W$, when we collapse the components of $\overline{M-N(W)}$. Foliations carried by $W$ may differ structurally, but each is transverse to the flow $\phi$. Accordingly, each may be used with $\phi$ and the generating set to construct $W$.

Let $p$ be a continuous surjective map from a branched surface $W^{\wedge}$ with charts $\Psi^{\wedge}$ onto a branched surface $W$ with charts $\Psi$. An open set $U$ of $W$ is "evenly covered" if the inverse image $p^{-1}(U)$ can be written as a union of disjoint open sets

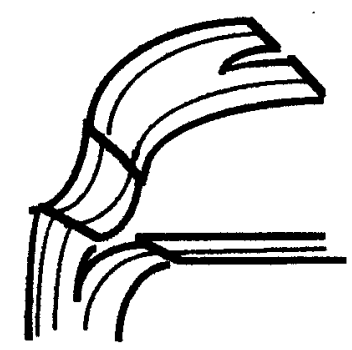

Figure 2.5. An example of how a foliation of $N\left(W_{\varepsilon}\right)$ appears locally. 


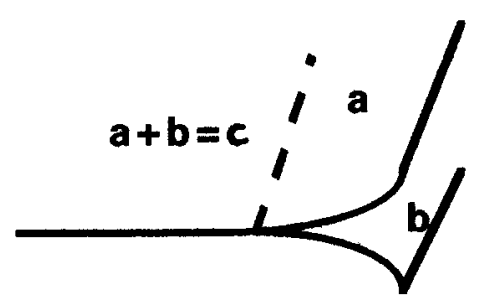

FiguRe 2.6

$V_{\alpha}$ in $W^{\wedge}$ such that for each $\alpha$, the restriction of $p$ to $V_{\alpha}$ is a diffeomorphism onto $U$ that maps right (left, respectively) crossings in $W^{\wedge}$ onto right (left, respectively) crossings in $W$. If every point in $W$ has an evenly covered neighborhood and each element of the composition $\Psi * p * \Psi^{\wedge-1}$ is smooth and orientation preserving, then $p$ is called a "covering map". In this case, we say " $W^{\wedge}$ covers $W$ ".

In $\S \mathrm{IV}$ we show that if $W$ carries a foliation $f$ and $f^{\wedge}$ is a covering of $f$, then there exists a covering of $W$ by a branched surface $W^{\wedge}$ carrying $f^{\wedge}$. We also show that if $W^{\wedge}$ is a branched surface covering $W$, then every foliation carried by $W$ is covered by a foliation carried by $W^{\wedge}$.

In [3], Floyd and Oertel define a weight system on a branched surface as an assignment of a nonnegative integer, called a "weight", to each sector in such a way that these numbers satisfy an obvious additive condition across any connected subset of the branch set that does not contain crossings (see Figure 2.6).

If $W$ is a compact branched surface, there are a finite number of crossings of the branch set $B$. The regular neighborhood $W_{\varepsilon}$ of $B$ in $W$ can be constructed by choosing a regular neighborhood $W_{i}$ of $B$ in a neighborhood of each crossing and using the transition maps to define appropriate pairwise identifications of these $W_{i}$ 's along the Y-shaped components of their boundaries. If $W$ has all disk sectors, we find the weight systems for $W$ by first assigning a variable to each connected component of $W_{i}-\left(B \cap W_{i}\right)$ for every $i$. The additive condition across the branch set, together with the identifications used to construct $W_{\varepsilon}$ from the $W_{i}$ 's, yields a finite homogeneous system of linear equations with integer coefficients. The weight systems on $W$ are given by the solutions to this system that have all nonnegative integral entries.

To find the weight systems for a compact $W$ with any type of sector, we assign variables to the sectors of $W$ and obtain a finite homogeneous system of linear equations with integer coefficients which is generated by the additive condition on weights across the branch set. The weight systems on $W$ are given by the solutions to this system that have all nonnegative integral entries. However, since $W_{\varepsilon}$ is easier to depict than $W$, this method is not as practical for $W$ with disk sectors.

For example, the Reeb foliation of $S^{3}$ is carried by a branch surface $W$ with disk sectors. The labeled graph of the branch set of $W$ is shown at left in Figure 2.7. A regular neighborhood of the branch set in a local neighborhood of the crossing is shown at right in Figure 2.7. The local conditions satisfied by the weights are indicated. The pairs of boundary components that are identified to construct $W_{\varepsilon}$ from this neighborhood are labeled "A" and "B".

Each branched surface has a trivial weight system where every weight is zero. Floyd and Oertel show that if a compact branched surface $W$ has a nontrivial weight system, then this weight system may be used to construct a compact surface 

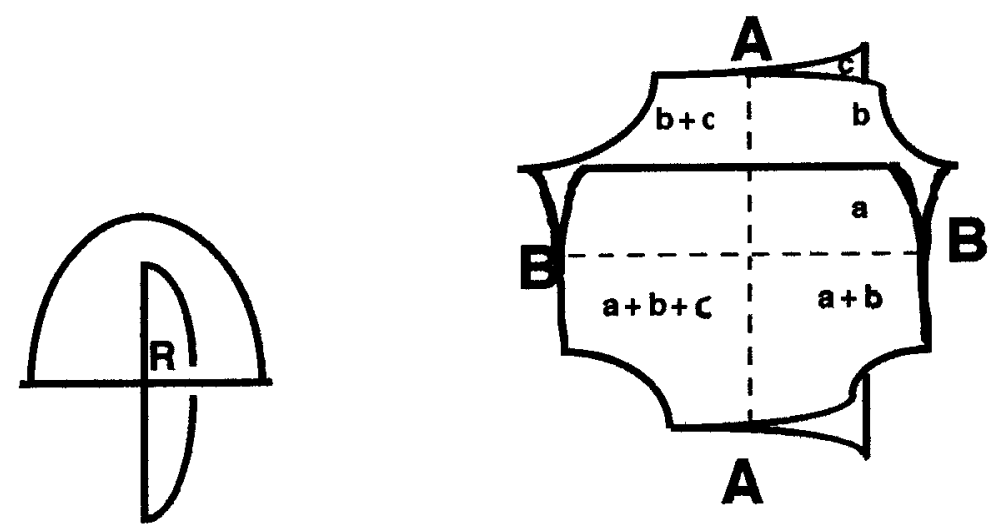

Figure 2.7. Identification A yields the equation $\mathbf{b}=\mathbf{a}+\mathbf{b} \Rightarrow \mathbf{a}=$ 0 . Identification $\mathbf{B}$ yields the equation $\mathbf{b}=\mathbf{b}+\mathbf{c} \Rightarrow \mathbf{c}=0$. So the weight systems on $W$ are (where $\mathbf{b}$ is a nonnegative integer)

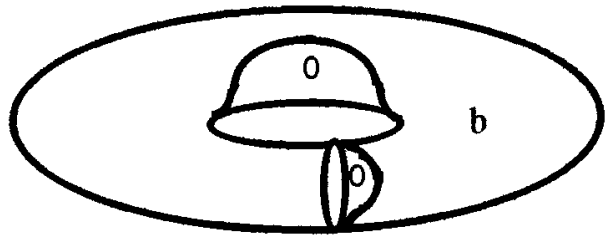

imbedded in $N(W)$ which "induces" the weights. For this we begin with parallel copies of each sector imbedded transverse to the fibers, where the number of copies of a given sector equals the weight of that sector. By the additive condition on weights, these copies may be pieced together over the branch set to form a compact surface $C$. Clearly, we may construct $C$ in such a way that it does intersect $\partial N(W)$. The weight system on $W$ is induced by $C$ by letting the weight of a sector be the number of times $C$ intersects a fiber over that sector.

Given the weighted branched surface in Figure 2.7, the restriction of a corresponding imbedded surface $C$ to $N\left(W_{\varepsilon}\right)$ is indicated in Figure 2.8.

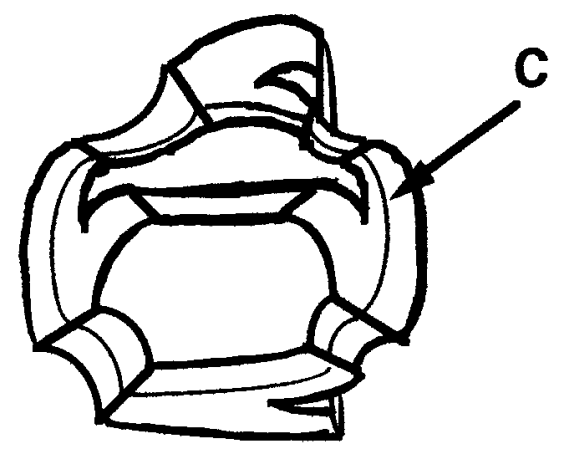

Figure 2.8 
If $W$ has a nontrivial weight system, there exists a foliation of $N\left(W_{\varepsilon}\right)$ for which the restriction of the imbedded surface $C$ corresponding to $N\left(W_{\varepsilon}\right)$ is a leaf. If we can extend this foliation to a foliation of $N(W)$ containing $C$, then we may collapse $M-N(W)$ to obtain a foliation of $M$ with a compact leaf. Conversely, if $f$ is a foliation of $N(W)$ with a compact leaf $C$ which does not intersect $\partial N(W)$, then $C$ induces a weight system on $W$.

\section{FiBRATIONS OVER $S^{1}$ AND POSITIVELY WEIGHTED BRANCHED SURFACES}

We now show that a compact branched surface $W$ has a weight system that assigns only positive weights if and only if it carries a fiber bundle over $S^{1}$. It will follow from the proof that we may use the strictly positive weight system on $W$ to identify the fiber. If the genus of this fiber is not zero, the fibration is covered by a trivial product of hyperplanes. In $\S \mathrm{IV}$, we find a sufficient condition on the covering of $W$ to guarantee that every foliation carried by $W$ is covered by a trivial product of hyperplanes. If the fiber is a sphere, we know by Reeb stability that every foliation carried by $W$ is a trivial fibration over $S^{1}$ by spheres ([12]).

Theorem 3.1. A compact branched surface $W$ carries a fibration over $S^{1}$ if and only if there exists a strictly positive weight system on $W$.

Proof. Suppose $W$ is assigned a strictly positive weight system. We may assume, using a change of metric if necessary, that the fibers over sectors of weight a have length a. Because of the additive condition across the branch set, we may also assume that the fibers over each portion of the branch set, where locally sectors of weight $\mathbf{a}$ and $\mathbf{b}$ are tangent to a third of weight $\mathbf{a}+\mathbf{b}$, have length $\mathbf{a}+\mathbf{b}$.

For each sector $S$, we foliate its fiber neighborhood $N(S)$ by a stack of leaves which meet each fiber at a fixed distance from the lower endpoint. (Here we assume a fiber's lower endpoint is determined by its orientation, and distance is measured along the fibers.) So every leaf in the foliation of a sector neighborhood $N(S)$ lies at a fixed distance from some component of $\partial N(W)$. We may construct a foliation of $N(W)$ by extending the foliations of the sector neighborhoods to fibers over the branch set in a manner that preserves the fixed distance of the leaves from these components of $\partial N(W)$. This gives a foliation $f^{*}$ of $N(W)$ with all leaves compact. When we collapse the complement of $N(W)$ to obtain $M, f^{*}$ yields a foliation $f$ of $M$ with all leaves compact. It follows that $M$ fibers over $S^{1}$ and $W$ carries this fibration.

Conversely, if $W$ carries a $F$-bundle over $S^{1}$ for some compact surface $F$, then each of the nonbranched leaves in the corresponding foliation of $N(W)$ is compact and homeomorphic to $F$. We may choose a finite set $\left\{F_{i}\right\}_{i=1}^{n}$ of leaves so that for any sector $S$ of $W$, its fiber neighborhood $N(S)$ is met by some leaf in this set. We then let the number of times a fiber over $S$ intersects $\bigcup\left\{F_{i}\right\}_{i=1}^{n}$ be the weight assigned to $S$. In this manner, we obtain a strictly positive weight system on $W$.

We note that the proof of Theorem 3.1 is constructive. If we use a positive weight system on $W$ to construct a foliation of $N(W)$ with all leaves compact, then any nonbranched leaf is a copy of the fiber in the corresponding fibration of the manifold $M$. In [10], Plante presents a result due to Gabai which states that if the genus of this fiber is greater than one, the foliation of $M$ by fibers is $C^{0}$-unstable. Foliations with no compact leaves are constructed arbitrarily close (according to 
the $C^{0}$ metric in [6]) to the fibration. The branched surface $W$ can be shown to carry at least one of these foliations. So we have the following:

Proposition 3.2. If a compact branched surface $W$ has a strictly positive weight system, then either there exists a foliation carried by $W$ with no compact leaves, or $M$ is a torus or sphere bundle over $S^{1}$.

Other results concerning the stability of compact leaves in foliations of fiber bundles are given in [7] and [10].

Our method for using a nonbranched compact leaf $C$ in a foliation of $N(W)$ to weight $W$ can be described as letting the holonomy invariant measure on the foliation be the Dirac measure concentrated on $C$ and defining the weight of each sector to be the measure of a fiber over that sector. Using a generalization of this method, it can be shown that a holonomy invariant measure for a foliation carried by $W$ that is finite on transverse arcs induces a weight system on $W$ ([13]). So the next result follows directly from Theorem 3.1. This result is well known for manifolds of any dimension, and an earlier proof can be found in [11].

Corollary 3.3. Let $v$ be a holonomy invariant measure for a codimension one foliation of a closed 3-manifold $M$. If the support of $v$ is $M$ and $v$ is finite on compact arcs, then $M$ is a fibration over $S^{1}$.

\section{Stability of Foliations COVERED By a trivial PRODUCT OF HYPERPLANES}

As above, we consider only those branched surfaces that can be constructed from transversely orientable codimension one $C^{1}$ foliations of Riemannian 3-manifolds without boundary using the technique described by Christy and Goodman. Consequently, we assume each branched surface $W$ is imbedded in a 3-manifold $M$ and is transversely oriented. We also assume the transverse orientation of $W$ and the orientation of the fibers of $N(W)$ are induced by the transverse flow used to construct $W$.

In what follows, the only product foliations we consider are topologically equivalent to the canonical foliation of $\mathfrak{R}^{3}$ by parallel hyperplanes. We will not consider other products such as the Reeb foliations of the product of $\mathfrak{R}$ and the sphere. We find a sufficient condition on a branched surface carrying a product foliation to ensure that all foliations it carries are topologically equivalent. We use this to find a condition guaranteeing that the property of being covered by a trivial product of hyperplanes is stable under $C^{1}$ perturbations. The results hold for branched 1-manifolds, and since imbeddings of branched 1-manifolds are easier to depict than the imbeddings of branched surfaces, we will sometimes illustrate results with lower-dimensional examples that have higher-dimensional analogs.

As noted in $\S I I$, every foliation carried by a branched surface $W$ uniquely corresponds to a foliation of $N(W)$. In order to avoid cumbersome notation, we use the same symbol to denote both foliations. It will be clear from the context to which foliation we are referring. Recall that $W$ is an imbedded copy of a quotient space $N(W) / \sim$ in $M$, where $x \sim y$ if $x$ and $y$ lie on the same fiber. Throughout, the map $\pi: N(W) \rightarrow W$ will be the composition of the quotient map with the imbedding. We may think of $\pi$ as the map that projects points along the fibers of $N(W)$ onto points in $W$. At each branching of a leaf $L$ there is a component of $\partial N(W)$ contained in $L$. We may restrict $L$ so that it contains only one hemisphere of this 
component. After restricting $L$ at each of its branchings, we have a nonbranched surface in $N(W)$ that yields the same leaf as $L$ when we collapse $M-N(W)$. It is important to note that whenever we take an image under $\pi$ of a branched leaf $L$, we first restrict $L$ is restricted in this manner.

Definition. An integral curve of a foliation is a curve that is contained in a leaf. Given a branched surface $W$ carrying a foliation $f$, an $f$-curve is the image under $\pi$ of an integral curve of $f$ (i.e., $\gamma$ is an $f$-curve if an integral curve of $f$ lies over $\gamma$ in $N(W)$ ). An $f$-loop is an $f$-curve that begins and ends at the same point. An $f$-surface is the image under $\pi$ of a leaf of $f$. A $W$-surface is an $f$-surface for some foliation $f$ carried by $W$. A $W$-curve is defined similarly.

Definition. Let $f$ be a foliation carried by $W$ and $\Gamma$ a curve of the form $\cdots * \alpha_{j+k} *$ $\beta_{j+k} * \cdots * \alpha_{k} * \beta_{k} * \cdots$ (possibly a finite composition), where each $\alpha_{i}$ is contained in a smooth surface in $W$ and has nonempty interior, and each $\beta_{i}$ is a transverse curve to $W$ with an orientation consistent with the transverse orientation on $W$. Given an $f$-surface $S$, we say the intersection of a composant $\beta_{i}$ of $\Gamma$ with $S$ is proper if $S \cap \beta_{i}$ is a single point contained in the interior of $\beta_{i}$. We say the intersection of a composant $\alpha_{i}$ of $\Gamma$ with $S$ is proper if one of the following holds:

i) $S$ meets both the beginning and the end of $\alpha_{i}$;

ii) $S$ meets only the beginning of $\alpha_{i}$ and branches away from $\alpha_{i}$ along a lower outgoing sector;

iii) $S$ meets only the end of $\alpha_{i}$ and branches away from $\alpha_{i}^{-1}$ along an upper outgoing sector.

The curve $\Gamma$ meets $S$ properly with respect to $f$ if:

1) any leaf of $f$ lying over $S$ intersects the fibers over $\Gamma \cap S$ exactly once,

2) $S-\left\{\alpha_{i} \cap S\right\}$ does not meet both a lower sector and an upper sector branching from $\alpha_{i}$ for any $i$

3) $S \cap \operatorname{int}\left(\beta_{i}\right)$ contains at most one point for each $i$ and

4) there is precisely one composant of $\Gamma$ whose intersection with $S$ is proper.

The curve $\Gamma$ is a global transversal for $f$ if $\Gamma$ meets every $f$-surface properly with respect to $f ; \Gamma$ is a global transversal for $W$ if it is a global transversal for every foliation carried by $W$.

Lemma 4.1. If $\Gamma$ is a global transversal for $W$, then for any foliation of $N(W)$ there is a homeomorphic copy of $\Gamma$ that meets every leaf transversely. Further, for any foliation carried by $W$, this copy becomes a homeomorphic imbedding of the leaf space when we collapse the complement of $N(W)$ in $M$.

Proof. Suppose $\Gamma$ is a global transversal for $W$ and $f$ is any foliation carried by $W$. By definition, $\Gamma$ is of the form $\cdots * \alpha_{j+k} * \beta_{j+k} * \cdots * \alpha_{k} * \beta_{k} * \cdots$ (possibly a finite composition), where each $\alpha_{i}$ is a curve in $W$ from the end of $\beta_{i}$ to the beginning of $\beta_{i+1}$, and each $\beta_{i}$ is transverse to $W$. We construct a copy $\beta_{i}^{\prime}$, transverse to $\partial N(W)$, of each $\beta_{i}$ by extending $\beta_{i}$ at its intersections with $W$ to include the fibers over these intersections. The curve $\beta_{i}^{\prime}$ meets all leaves that are mapped by $\pi$ onto an $f$-surface $S$ where $\beta_{i} \cap S$ is proper. Now suppose $\beta_{i}$ has a positive boundary point and $\beta_{i+1}$ has a negative boundary point. Consider the fiber $I_{i}$ over the end of $\beta_{i}$ and the fiber $I_{i+1}$ over the beginning of $\beta_{i+1}$. We modify $\beta_{i}^{\prime} \cap I_{i}$ and $\beta_{i+1}^{\prime} \cap I_{i+1}$, staying transverse to the leaves of $f$, to obtain a single curve over $\alpha_{i}$ which begins at the lower end of $I_{i}$ and ends at the upper end of $I_{i+1}$. This modification can 


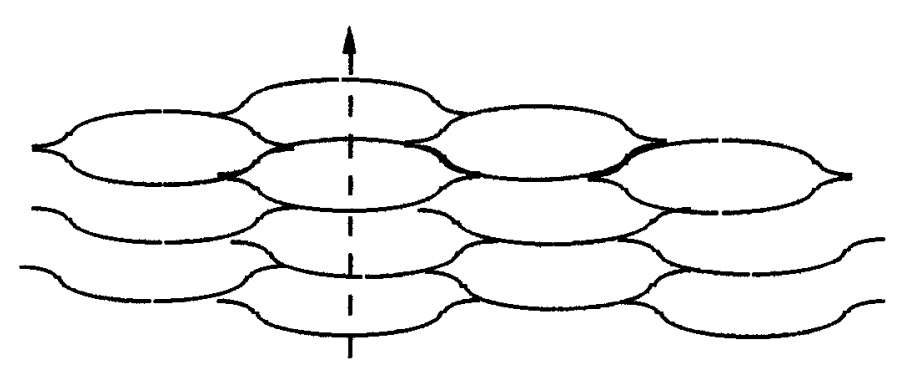

Figure 4.1

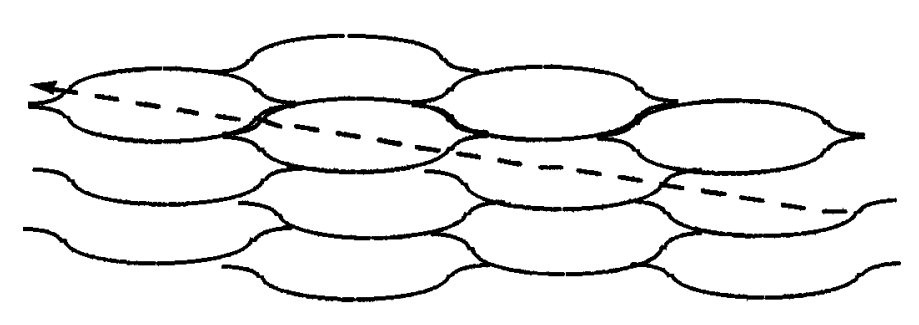

FiguRE 4.2

be chosen to meet precisely those leaves that are mapped by $\pi$ onto an $f$-surface $S$ where $\alpha_{i} \cap S$ is proper, since $S-\left\{\alpha_{i} \cap S\right\}$ does not meet both a lower sector and an upper sector branching from $\alpha_{i}$. If some $\alpha_{i}$ is noncompact, then we similarly modify the fiber over its boundary so it is transverse to the leaves of $f$ and lies over $\alpha_{i}$. The curve $\Gamma$ meets every $f$-surface properly, so after all these modifications of $\Gamma$ we have a transverse copy of $\Gamma$ that meets every leaf of $f$ exactly once.

Since the orientation for each $\beta_{i}^{\prime}$ is consistent with the transverse orientation on $\partial N(W)$, each intersection of $\beta_{i}^{\prime}$ with a component of $M-N(W)$ is an arc extending from the lower hemisphere to the upper hemisphere of the boundary of this component. We may collapse the components of $M-N(W)$ which are met by $\beta_{i}^{\prime}$ so that points in the same arc in $\beta_{i}^{\prime} \cap \overline{M-N(W)}$ are identified. Therefore, we may collapse the components of $\overline{M-N(W)}$ so that the copy of $\Gamma$ constructed above becomes an imbedding of the leaf space for $f$ in $M$.

If a foliation $f^{\wedge}$ of $\mathfrak{R}^{3}$ has leaf space $\mathfrak{R}$ and covers a foliation $f$ of a closed manifold, then all leaves of $f^{\wedge}$ are planes. We may use Lemma 4.1 to conclude that for $W^{\wedge}$ imbedded in $\mathfrak{R}^{3}$, if $W^{\wedge}$ has a global transversal and covers a compact $W$, then every foliation carried by $W^{\wedge}$ that covers a foliation carried by $W$ is a trivial product of hyperplanes.

Figure 4.1 shows a curve $\Gamma$ on a branched 1-manifold $W^{\wedge}$ imbedded in $\mathfrak{R}^{2}$ that is a global transversal for $W^{\wedge}$. This $W^{\wedge}$ covers a branched 1-manifold $W$ imbedded in a torus. When we thicken $W^{\wedge}$ to recover $N\left(W^{\wedge}\right), \Gamma$ yields a curve which meets the leaves in any foliation of $N\left(W^{\wedge}\right)$ exactly once. When we collapse the complement of $N\left(W^{\wedge}\right)$ in $\mathfrak{R}^{2}$, this curve yields a homeomorphic copy of $\mathfrak{R}$. So the leaf space of every foliation carried by $W^{\wedge}$ is $\mathfrak{R}$, as guaranteed by Lemma 4.1. Now, every foliation carried by $W$ is covered by a foliation carried by $W^{\wedge}$, so each is covered by a trivial product of hyperplanes. Figure 4.2 shows a curve on $W^{\wedge}$ that is not a global transversal for $W^{\wedge}$. 


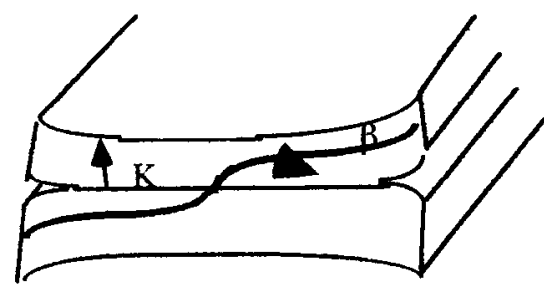

Figure 4.3. A curve $\beta$ which crosses the connecting strip $K$.

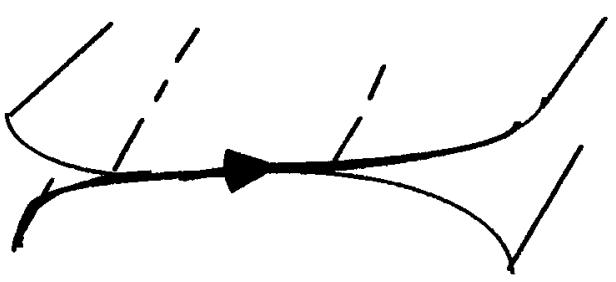

Figure 4.4. Its projection under $\pi$ crossing the corresponding connecting curve in increasing order.

Before stating Lemma 4.2 we give several definitions and review some results from [13] which are easily verified.

A curve $\kappa(t)_{0 \leq t \leq 1}$ in $W$ is a connecting curve of $W$ if there are two incoming sectors branching from $\kappa$ at $\kappa(0)$ and two outgoing sectors branching from $\kappa$ at $\kappa(1)$. The image $K$ of an immersion $\kappa^{*}:[0,1] \times[-\varepsilon, \varepsilon] \rightarrow N(W)$ is a connecting strip of $N(W)$ if:

1) only its ends, $\kappa^{*}(\{0\} \times[-\varepsilon, \varepsilon])$ and $\kappa^{*}(\{1\} \times[-\varepsilon, \varepsilon])$, are contained in the set of furrow points for $N(W)$,

2 ) it is transverse to the fibers, and

3) the image of the curve $\left.\kappa^{*}(t, 0)\right|_{0 \leq t \leq 1}$ under $\pi$ is a connecting curve $\kappa$.

So for each connecting strip $K$ there exists a corresponding connecting curve $\kappa$. We shall consider only those connecting curves in $W$ that correspond to some connecting strip.

Suppose points in the same fiber of $N(W)$ are ordered according to the orientation of the fiber. Let $\alpha$ be a curve transverse to the fibration $N(W)$ over $W$. (We note that this is a slight abuse of terminology, since $N(W)$ is not quite a fibration.) Suppose its projection $\pi(\alpha)$ is the connecting curve $\kappa$, and let $\beta$ be any curve containing $\alpha$. If $\alpha$ begins at a point below or contained in $\partial K$ (where "above" and "below" are determined by the orientation of the fiber over $\kappa(0))$ and ends at a point above or contained in $\partial K$, then $\pi(\beta)$ crosses $\kappa$ in increasing order. (See Figures 4.3 and 4.4.) If $\alpha$ begins at a point above or contained in $\partial K$ and ends at a point below or contained in $\partial K$, then $\pi(\beta)$ crosses $\kappa$ in decreasing order. So if $\alpha$ begins and ends in $\partial K$, then $\pi(\beta)$ crosses $\kappa$ in both orders.

For the remainder of this section, $f$ is a foliation that is covered by a trivial product of hyperplanes $f p$. Further, the curve $\Gamma^{*}$ will be an oriented imbedding of the leaf space of $f p$. We note the construction of an important branched surface covering $W$ when $W$ carries the foliation $f$. Suppose $\phi$ is the transverse flow to $f$ used in the construction of $W$, and let $\phi^{\wedge}$ be the lift of $\phi$. Take all the disks in the generating set of $W$ and use their lifts, together with $\phi^{\wedge}$, to construct a branched surface $W^{\wedge}$ from $f p$. (It is easy to verify that these lifted disks satisfy the general position requirements for a generating set.) Clearly $N\left(W^{\wedge}\right)$ covers $N(W)$ in such a way that fibers cover fibers. It follows that $W^{\wedge}$ covers $W$. So, given a branched surface $W$ carrying the foliation $f$, we may always assume the existence of a branched surface covering $W$ and carrying $f p$. For the remainder of this section, 
$W^{\wedge}$ will represent this branched surface, and we assume it was constructed as above. We let $\Gamma$ be the curve induced by $\Gamma^{*}$ when we construct $W^{\wedge}$ (i.e., $\Gamma$ will be the image of $\Gamma^{*}$ after we cut open along the disks in the generating set for $W^{\wedge}$ and collapse the fibers of $N\left(W^{\wedge}\right)$ ). We may assume (changing the orientation of $\Gamma^{*}$ if necessary) that every place $\Gamma$ is transverse to $W^{\wedge}$ the orientation of $\Gamma$ is consistent with the transverse orientation of $W^{\wedge}$. So $\Gamma$ is of the form $\cdots * \alpha_{j+k} * \beta_{j+k} * \cdots * \alpha_{k} * \beta_{k} * \cdots$ (possibly a finite composition), where each $\alpha_{i}$ is a curve in $W^{\wedge}$ and each $\beta_{i}$ is a curve positively transverse to $W^{\wedge}$. Now $\pi: N\left(W^{\wedge}\right) \rightarrow W^{\wedge}$ is one-to-one on leaves in $f p$, since when we cut $\mathfrak{R}^{3}$ open along disks to obtain $N\left(W^{\wedge}\right)$, the fibers are induced by the orbits of the transverse flow. It is straightforward to verify that $\Gamma^{*}$ can be chosen to ensure $\Gamma$ is a global transversal for $f p$, so we assume this to be the case.

If there is a $W^{\wedge}$-curve $\alpha^{\wedge}$ that is not an $f p$-curve, then clearly $\alpha^{\wedge}$ covers a curve $\alpha$ in $W$ that is not an $f$-curve. We see that $\alpha$ contains some connecting curves, called critical $f$-curves, which are crossed by $f$-curves and by $\alpha$, but not in the same order $([13])$.

We now restrict our attention to a type of $W$-curve $\alpha$ and the critical $f$-curves it contains.

Definition. If $\alpha$ is a $W$-curve and some curve $\alpha^{\wedge}$ covering $\alpha$ is contained in a $W^{\wedge}$-surface that is not properly met by $\Gamma$, then any critical $f$-curve $\kappa$ in $\alpha$ is a weak connecting curve in $W$ with respect to $(f, \Gamma)$.

In the following lemma we show that if $\Gamma$ is not a global transversal for $W^{\wedge}$, then there exists a weak connecting curve $\kappa$. By definition, $\kappa$ is a connecting curve in $W$ which is crossed by $f$-curves, but not in the same order as it is crossed by $\alpha \supseteq \kappa$. This will be significant for Theorem 4.3.

Lemma 4.2. If $\Gamma$ is not a global transversal for the branched surface $W^{\wedge}$ covering $W$, then every $W^{\wedge}$-surface not properly met by $\Gamma$ contains a $W^{\wedge}$-curve that is not an $f p$-curve.

Proof. Assume $\Gamma$ is not a global transversal for $W^{\wedge}$. Let $f^{\prime}$ be a foliation carried by $W^{\wedge}$ and $S^{\prime}$ be an $f^{\prime}$-surface that is not properly met by $\Gamma$ with respect to $f^{\prime}$. If some leaf of $f^{\prime}$ over $S^{\prime}$ intersects a fiber more than once, then there is an integral curve $\gamma$ of $f^{\prime}$ that lies over $S^{\prime}$ and connects two points in this intersection. If $\gamma$ projects onto an $f p$-loop in $W^{\wedge}$, then this $f p$-loop is the projection of an integral loop of $f p$; so it is null homotopic in $W^{\wedge}$. In this case, there is an integral loop in $f^{\prime}$ with the same projection ([13]) and this loop is null homotopic in a leaf of $f^{\prime}$. But this loop has nontrivial holonomy along its boundary, contradicting Reeb stability ([12]). So the projection $\alpha^{\wedge}$ of $\gamma$ is an $f^{\prime}$-loop, but is not an $f p$-loop.

Now, assume no leaf of $f^{\prime}$ over $S^{\prime}$ intersects a fiber more than once. If $S^{\prime}$ is not the union of $f p$-surfaces, then it contains a $W^{\wedge}$-curve $\alpha^{\wedge}$ that is not an $f p$-curve ([14]). So suppose $S^{\prime}$ is a union of $f p$-surfaces. If for some composant $\alpha_{i}$ of $\Gamma$, $S^{\prime}-\left\{\alpha_{i} \cap S^{\prime}\right\}$ intersects both an upper sector and a lower sector branching from $\alpha_{i}$, then any curve $\alpha^{\wedge}$ in $S^{\prime}$ which connects these intersections is not an $f p$-curve. So we consider the case where for each $i, S^{\prime}-\left\{\alpha_{i} \cap S^{\prime}\right\}$ meets only lower sectors or only upper sectors branching from $\alpha_{i} \subseteq \Gamma$. Since every $f p$-surface contained in $S^{\prime}$ is properly intersected by some composant of $\Gamma$, each of these composants properly intersects $S^{\prime}$. In fact, at least two distinct composants of $\Gamma$ properly intersect $S^{\prime}$ since $S^{\prime}$ is not properly met by $\Gamma$. There is an $f^{\prime}$-curve $\alpha^{\wedge}$ in $S^{\prime}$ that contains every 
component of these two proper intersections. Clearly $\alpha^{\wedge}$ is not an $f p$-curve (or the $f p$-surface containing it would not be properly met by $\Gamma$ ).

We say two connecting curves $\kappa$ and $\kappa^{\prime}$ contained in $W$ are equivalent if $\kappa$ and $\kappa^{\prime}$ correspond to the same connecting strip. All references to connecting curves in $W$ will, in fact, refer to the equivalence class unless otherwise stated.

Suppose $W$ is constructed from the foliation $f$. For the following discussion, we let $f^{*}$ be the corresponding foliation of $N(W)$ to avoid confusion. Let $\kappa$ be any connecting curve of $W$ that begins at a branch point $\partial^{\prime} \in W$, and let $\partial$ be the furrow point in $\partial N(W)$ over $\partial^{\prime}$. We show how to construct a new branched surface for which $\kappa$ is not a connecting curve whenever $f^{*}$ has an integral curve through $\partial$ that lies over $\kappa$. For simplicity, we will assume the generating set $\Delta$ for $W$ is a set of imbedded disks; however, the construction works if $\Delta$ is a collection of imbedded compact planar surfaces. First we note that when we collapse the components of $M-N(W)$ to give $f, \partial$ is identified with a point in the boundary of some $D \in \Delta$. The integral curve through $\partial$ lying over $\kappa$ is then identified with an integral curve of $f$ beginning in $\partial D$. We modify $\Delta$ by extending $D$ so it contains this integral curve of $f$ and still lies in a leaf of $f$ (possibly creating a different subset of the plane). We construct a new branched surface $W^{\prime}$ from $f$, for which $\kappa$ is not a connecting curve, using $f$ with the same transverse flow and the modified generating set. Since the effect of modifying the generating set for $W$ in this manner is to split $W$ along a smooth surface containing $\kappa$, we say the connecting curve $\kappa$ has been deleted by a "splitting in $f$ ". Since the extended $D$ may not be an imbedded disk, it is important (for Theorem 4.3) to note that Lemmas 4.1 and 4.2 did not rely on the assumption that the generating set for the branched surface consists only of imbedded disks. It is also worth noting that if some curve $\alpha$ crosses $\kappa$ in a different order than it is crossed by $f$-curves (e.g. $\kappa$ is weak in $W$ with respect to $(f, \Gamma)$ ), then $\alpha$ is not a $W^{\prime}$-curve.

The upper figure in Figure 4.6 shows a portion of the branched surface carrying $f$. Its fiber neighborhood is depicted in Figure 4.5. The lower figure is the corresponding portion of the new branched surface we have after the connecting curve is deleted by the splitting in $f$.
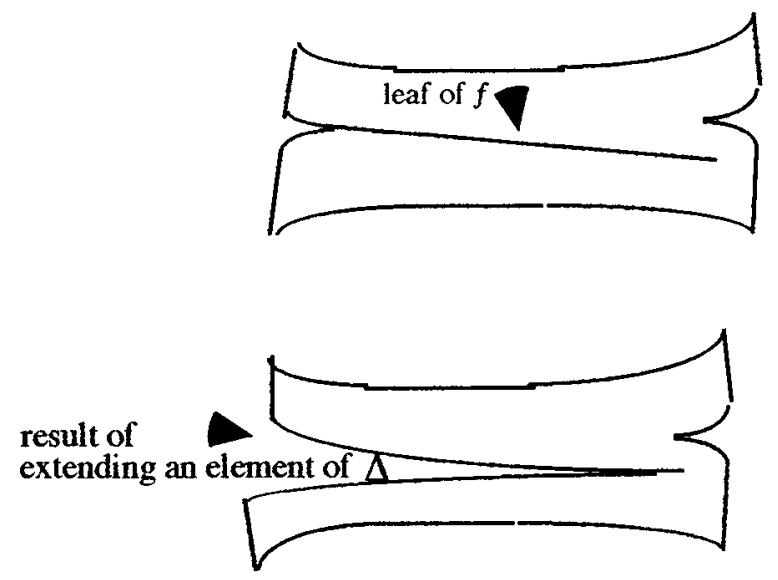

FiguRE 4.5 


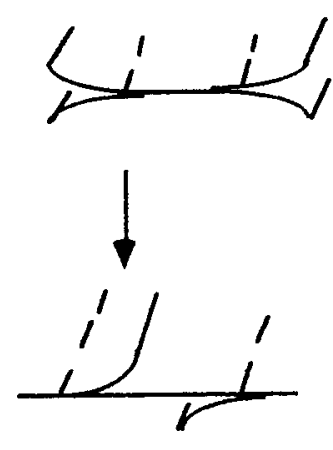

Figure 4.6

Since $f$ is covered by the product foliation $f p$, the branched surface $W^{\prime}$ is covered by a branched surface $W^{\prime \wedge}$ carrying $f p$. Let $\Gamma^{\prime}$ be the curve induced by the leaf space $\Gamma^{*}$ during the construction of $W^{\prime \wedge}$. Recall that $\Gamma$ is the curve induced by $\Gamma^{*}$ during the construction of $W^{\wedge}$. It is worth noting that $\Gamma^{\prime}$ can be slightly modified so that each $W^{\prime \wedge}$-surface not properly met by $\Gamma^{\prime}$ is also a $W^{\wedge}$-surface not properly met by $\Gamma$. We will always assume $\Gamma^{\prime}$ has this property.

Theorem 4.3. Let $f$ be a foliation of a compact manifold that is covered by a trivial product of hyperplanes. Suppose $W$ carries $f$, and the deletion of a finite number of connecting curves in $W$ by splittings in $f$ yields a branched surface $W^{\prime}$ for which there are no weak connecting curves with respect to $\left(f, \Gamma^{\prime}\right)$. Then $W^{\prime}$ carries $f$, and every foliation carried by $W^{\prime}$ is covered by a trivial product of hyperplanes.

Proof. Suppose the deletion of the finite number of connecting curves in $W$ by extensions in $f$ yields a branched surface $W^{\prime}$ which has no weak connecting curves with respect to $\left(f, \Gamma^{\prime}\right)$. Then by Lemma $4.2, \Gamma$ is a global transversal for $W^{\prime \wedge}$. So, according to Lemma 4.1, every foliation carried by $W^{\prime \wedge}$ that covers a foliation carried by $W^{\prime}$ is a trivial product of hyperplanes. We need only show that every foliation carried by $W^{\prime}$ lifts to a foliation carried by $W^{\prime \wedge}$. This is equivalent to showing that every foliation of $N\left(W^{\prime}\right)$ is covered by a foliation of $N\left(W^{\prime \wedge}\right)$. Clearly, every foliation of $N\left(W_{\varepsilon}^{\prime}\right)$ lifts to a foliation of $N\left(W_{\varepsilon}^{\prime \wedge}\right)$. Consider the restriction of the covering $p$ to a disk in a sector of $W^{\prime \wedge}$. There is an induced map on the fundamental group, so disks in sectors $W^{\prime \wedge}$ can only cover disks in sectors of $W^{\prime}$, and in each case the cover must be a diffeomorphism. Thus, we can complete any lift of a foliation restricted to $N\left(W_{\varepsilon}^{\prime}\right)$ to a foliation of $N\left(W^{\prime \wedge}\right)$ which covers it. From this the result is immediate.

Consider the $C^{1}$ metric for foliations defined in [6] using the tangent bundles to the leaves. Given a branched surface $W$ constructed from a foliation of a compact manifold, all sufficiently small perturbations of the foliation are carried by $W$ ([13]). (This is not true for branched surfaces constructed from foliations of noncompact manifolds.) Combining this with Theorem 4.3, we now have:

Corollary 4.4. Let $f$ be a foliation of a compact manifold that is covered by a trivial product of hyperplanes. If $f$ is carried by a branched surface satisfying the hypothesis of Theorem 4.3, then there is an $\varepsilon>0$ so that every foliation within $\varepsilon$ of $f$ in the $C^{1}$ metric is also covered by a trivial product of hyperplanes. 


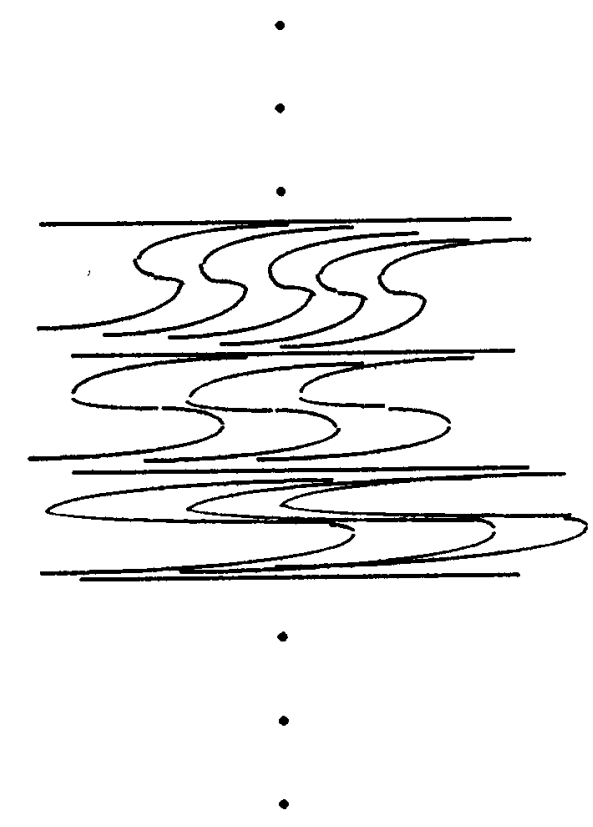

Figure 4.7. Example. This is a foliation of the Euclidean plane that is covered by a trivial product of hyperplanes. Arbitrarily close to this foliation (in the $C^{1}$ metric of [6]) we can find a foliation that contains two Reeb-like components which any transverse curve entering cannot leave. It follows that this nearby foliation is not covered by a trivial product of hyperplanes.

It may be possible to strengthen Theorem 4.3. Indeed, there may always exist a choice for the imbedding $\Gamma^{*}$ of the leaf space and the generating set $\Delta$ that ensures $W$ has no weak connecting curves with respect to $(f, \Gamma)$. However, the example in Figure 4.7, suggested by S. Goodman, shows the necessity of compactness of the manifold for stability of the property of being covered by the trivial product of hyperplanes.

In general, the most obvious branched surface carrying a foliation covered by a product contains no connecting curves, and in this case the hypothesis of Theorem 4.3 is trivially satisfied. In cases where $W$ contains weak connecting curves, it is worth noting that if a weak connecting curve $\kappa$ is deleted by a splitting in $f$, then any connecting curve contained in $\kappa$ that begins at the same point is also deleted. Problems satisfying the hypothesis of Theorem 4.3 arise when there is not a finite set of weak connecting curves that contains all others. This can occur when an infinite set of weak connecting curves share certain loops and differ only in the number of times these loops are traversed. In this case, there is no ordering of this set of curves by inclusion. In what follows, we find a condition on such a set that is sufficient to guarantee all its elements can be deleted by a single extension. We will let $\alpha^{n}$ represent the composition of a loop $\alpha$ with itself $n$ times.

Consider a connecting curve $\kappa$ of the form $\beta_{n+1} * \alpha_{n} * \beta_{n} * \cdots * \alpha_{1} * \beta_{1}$, where $\beta_{1}$ and $\beta_{n+1}$ both contian more than one point and each $\alpha_{i}$ is a simple loop. Suppose that whenever $\beta_{i+1}$ is a single point we have $\alpha_{i} \neq \alpha_{i+1}$. Let $\left(Z^{n}\right)^{+}$be a subset of 
$\mathfrak{R}^{n}$ consisting of $n$ vectors with only nonnegative integral entries, and let $z_{i}$ be the $i$ th entry in $z \in\left(Z^{n}\right)^{+}$. Now, $\beta_{n+1} * \alpha_{n}^{z_{n}} * \beta_{n} * \cdots * \alpha_{1}^{z_{1}} * \beta_{1}$ and $\beta_{n+1} * \beta_{n} * \cdots * \beta_{1}$ are also connecting curves. We denote $\beta_{n+1} * \alpha_{n}^{z_{n}} * \beta_{n} * \cdots * \alpha_{1}^{z_{1}} * \beta_{1}$ by $\kappa(z)$ (where the notation assumes the above decomposition of $\kappa$ ) and $\beta_{n+1} * \beta_{n} * \cdots * \beta_{1}$ by $\beta$.

In what follows, we use the notation $x<y$ if $x$ and $y$ are on the same fiber and $x$ is below $y$ with respect to the orientation of the fiber. Given a compact arc $\left.\gamma(t)\right|_{0 \leq t \leq 1}$ in $W$ and a point $x$ in the fiber over $\gamma(0)$, if there is an integral curve of $f$ that begins at $x$ and lies over $\gamma$, we let $h_{\gamma}(x)$ represent the end of this curve over $\gamma(1)$.

Definition. Let $\partial_{0}$ and $\partial_{1}$ be furrow points of $N(W)$ that lie in a connecting strip over the ends of the corresponding connecting curve $\kappa$. Suppose some integral curve begins at $\partial_{0}$ and lies over $\kappa$, and $\kappa$ has a decomposition $\beta_{n+1} * \alpha_{n} * \beta_{n} *$ $\cdots * \alpha_{1} * \beta_{1}$ as above such that $h_{\beta}\left(\partial_{0}\right) \leq \partial_{1}$. This decomposition is monotonic if $h_{\alpha_{k} * \beta_{k} * \cdots * \beta_{2} * \beta_{1}}\left(\partial_{0}\right) \leq h_{\beta_{k} * \cdots * \beta_{2} * \beta_{1}}\left(\partial_{0}\right)$ for every $1 \leq k \leq n$.

We assume the corresponding definition in the case where $h_{\beta}\left(\partial_{0}\right) \geq \partial_{1}$, obtained by reversing the transverse orientation.

The hypothesis of Theorem 4.3 is more likely to hold if every weak connecting curve is of the form $\kappa(z)$ for some $\kappa$ with a monotonic decomposition (and this is clearly dependent on $f$ ). For example, if $\beta_{2} * \alpha_{1} * \beta_{1}$ is a connecting curve such that $h_{\alpha_{1} * \beta_{1}}\left(\partial_{0}\right)>h_{\beta_{1}}\left(\partial_{0}\right), h_{\beta_{2} * \alpha_{1}^{n} * \beta_{1}}\left(\partial_{0}\right)<\partial_{1}$ for $n \geq 0$, and $\beta_{2} \alpha_{1}^{n} \beta_{1}$ is weak connecting with respect to $(f, \Gamma)$ for $n \geq M$, then when we delete $\beta_{2} \alpha_{1}^{M} \beta_{1}$ by extensions in $f$, we still have weak connecting curves $\beta_{2} \alpha_{1}^{n} \beta_{1}$ for $n>M$. In this case, the hypothesis of Theorem 4.3 is not satisfied.

In Proposition 4.5 we focus on a subset $K_{\Gamma}(f)$ of weak connecting curves with respect to $(f, \Gamma)$ that it is sufficient to delete; that is, if we delete all curves in $K_{\Gamma}(f)$ by splittings in $f$, then we obtain a branched surface $W^{\prime}$ with no weak connecting curves with respect to $\left(f, \Gamma^{\prime}\right)$, where $\Gamma^{\prime}$ is the curve induced in the construction of $W^{\prime \wedge}$ by the leaf space of the product $f p$. We let $K_{\Gamma}(f)=$ \{connecting curves which cannot be written as $\kappa(z)$ for any $\kappa\} \cup\{\kappa(z) ; \kappa$ is a connecting curve with a monotonic decomposition, $\kappa(z)$ is weak connecting, and $z \in\left(Z^{n}\right)^{+}$has at most one nonzero entry\}. It is tedious but straightforward to verify that, using splittings in $f$ to delete all curves in $K_{\Gamma}(f)$, we may construct a new branched surface $W^{\prime}$ for which there are no weak connecting curves with respect to $\left(f, \Gamma^{\prime}\right)$.

Proposition 4.5. If $K_{\Gamma}(f)$ is finite, then the hypothesis of Theorem 4.3 is satisfied.

We end this section with an example illustrating Theorem 4.3 and Proposition 4.5. We consider a branched surface $W$ that carries the foliation $f$ of $T^{2} \times S^{1}$ shown in Figure 4.8. The branch set for $W$ is depicted in Figure 4.9. (Since $W$ does not admit a strictly positive weight system, it does not carry a fibration.) The foliation $f$ is covered by a trivial product of hyperplanes. Figure 4.10 shows part of $W_{\varepsilon}$ which contains the three crossings on the left of Figure 4.9. The loop in Figure 4.10 is induced by a transverse loop to $f$ that lifts to a copy of the leaf space $\Gamma$. (The fine lines in this loop indicate arcs between the two boundaries of an annulus sector.) The arcs $\alpha_{0}$ and $\alpha_{1}$ in this loop are tangent to $W_{\varepsilon}$, and $\beta_{1}$ is transverse. The shaded region is contained in a $W$-surface whose lift is met twice by $\Gamma$. It can be verified that $K_{\Gamma}(f)$ contains only three curves. The first is indicated by the bold line on the graph of the branch set shown in Figure 4.9; it contains no simple loops. 


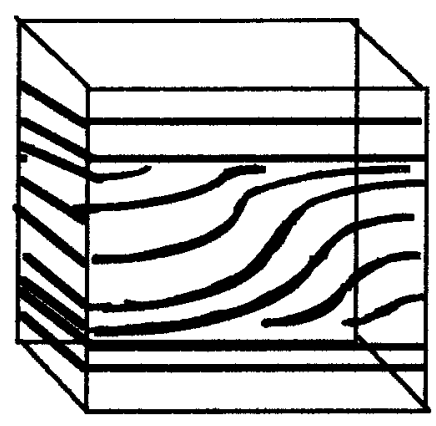

FIGURE 4.8. This foliation of the cube induces a foliation $f$ of $T^{2} \times S^{1}$ when opposite sides are glued by the identity.

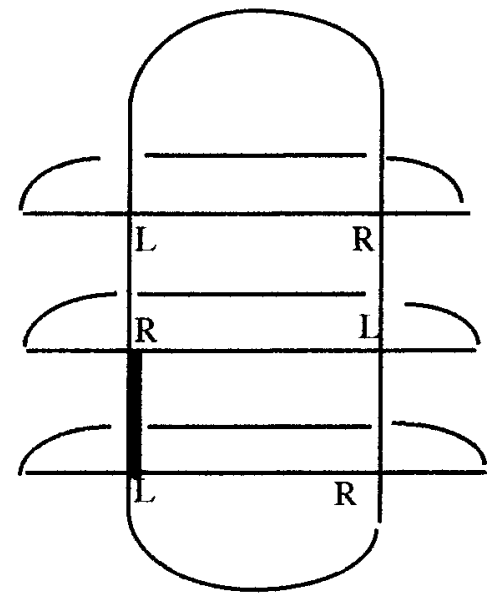

Figure 4.9

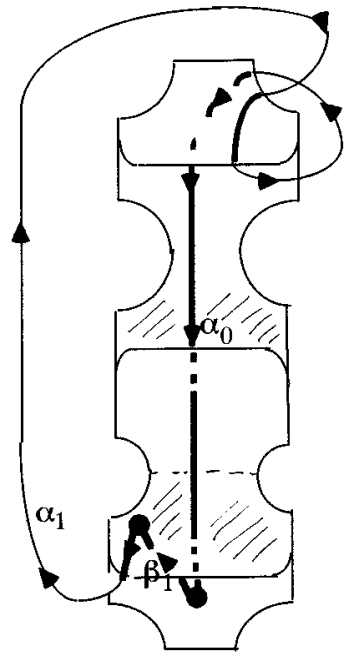

FiguRE 4.10

The other two differ from the first by a simple loop; both can be shown to have a monotonic decomposition. So, we may delete three connecting curves by extensions in $f$ to obtain a branched surface $W^{\prime}$ carrying $f$ where every foliation carried by $W^{\prime}$ is covered by a trivial product of hyperplanes. Therefore, every foliation $C^{1}$ close to $f$ is covered by a trivial product of hyperplanes.

\section{REFERENCES}

1. J. Christy and S. Goodman, Branched surfaces transverse to codimension one foliations, working paper, Emory Univ., Atlanta, GA, and University of North Carolina, Chapel Hill, NC, n.d.

2. J. Christy, Branched surfaces and attractors. I: Dynamic branched surfaces, Trans. Amer. Math. Soc. 336 (1993), 759-784. MR 93f:58135 
3. W. Floyd and U. Oertel, Incompressible surfaces via branched surfaces, Topology 23 (1984), 117-125. MR 85a:57007

4. J. Franks and R. F. Williams, Anomolous Anosov flows, Common Theory of Dynamical Systems, Lecture Notes in Math., vol. 819, Springer, Berlin, 1980, pp. 158-174. MR 82e:58078

5. J. Hempel, 3-manifolds Ann. of Math. Studies, vol. 86, Princeton Univ. Press, Princeton, NJ, 1976. MR 54:3702

6. Morris W. Hirsch, Stability of compact leaves of foliations, Dynamical Systems (Proc. Sympos., Salvador, Brazil, 1971; M. Peixoto, ed.), Academic Press, New York, 1973, pp. 135-153. MR 48:12555

7. R. Langevin and H. Rosenberg, On stability of compact leaves and fibrations, Topology 16 (1977), 107-112. MR 57:1508

8. S. P. Novikov, Topology of foliations, Trudy Moskov. Mat. Obschch. 14 (1965), 248-277; English transl., Trans. Moscow Math. Soc. 14 (1977), 268-303. MR 34:824

9. C. Palmeira, Open manifolds foliated by planes, Ann. of Math. (2) 107 (1978), 109-131. MR 58:18490

10. J. Plante, Stability of codimension one foliations by compact leaves, Topology 22 (1983), 173-177. MR 84g:57022

11. , Anosov flows, Amer. J. Math. 94 (1972), 729-754. MR 51:14099

12. G. Reeb, Sur certaines propriétés topologiques des variétées feuilletées, Actualités Sci. Indust., no. 1183, Hermann, Paris, 1952. MR 14:1113a

13. S. Shields, Branched surfaces and the stability of compact leaves, Thesis, University of North Carolina, Chapel Hill, NC, 1991.

14. , Branched surfaces and the simplest foliations of 3-manifolds, preprint.

15. A. Verjovsky, Codimension one Anosov flows, Bol. Soc. Math. Mexicana 19 (1974), 49-77. MR 55:4282

16. R. F. Williams, Expanding attractors, Inst. Hautes Études Sci. Publ. Math. No. 43 (1973), 169-203. MR 50:1289

Department of Mathematics, College of Charleston, Charleston, South Carolina 29424 